\title{
GÉNERO Y ETNICIDAD. SER HOMBRE Y SER MUJER ENTRE LOS AYMARA DEL ALTIPLANO CHILENO*
}

\author{
GENDER AND ETHNICITY. BEING MEN AND BEING WOMEN \\ AMONG THE AYMARA PEOPLE OF THE CHILEAN HIGHLANDS
}

\author{
Ana María Carrasco Gutiérrez ${ }^{* *}$ y Vivian Theda Gavilán Vega**
}

\begin{abstract}
En este artículo abordamos las relaciones de género, entre los aymara del norte de Chile, a partir del estudio de dos comunidades ubicadas en el altiplano de la Región de Tarapacá: Isluga y Cariquima. El interés central es conocer cómo se construye social y simbólicamente el género entre los aymara contemporáneos. Se indaga aspectos de las esferas social, económica y religiosa para entender la posición y condición de mujeres y hombres y las representaciones de lo femenino, lo masculino y sus relaciones. Este análisis de la vida social aymara permite vincular ciertas prácticas sociales a sus significados.
\end{abstract}

Palabras claves: aymara, género, etnicidad.

This article is about gender relations among the Aymara people of northern Chile, from the study of two communities in the highlands of the Tarapacá Region: Isluga and Cariquima. The main interest is to understand how gender is social and symbolically built among contemporary Aymara people. Aspects of social, economic and religious spheres are investigated to understand the position and status of women and men and also the representations of the feminine, masculine and their relationships. This analysis of the Aymara social life allows linking certain social practices and their meanings.

Key words: Aymara, gender, ethnicity.

\section{Introducción}

La dimensiones de género y étnica adquieren cada vez mayor relevancia para comprender las relaciones entre hombres y mujeres en espacios multiculturales como los países latinoamericanos, y entre estos Chile no es excepción.

Abordar el tema de género entre la población aymara es una tarea ambiciosa, pero creemos que los resultados aquí presentados pueden ser una guía de indagación que permita avanzar en la temática y entregar elementos interesantes para generar un proceso de reflexión que contribuya a un mayor conocimiento de nuestra realidad social y, así también, a una mejor convivencia entre sus actores.

$\mathrm{Al}$ investigar sobre las prácticas de hombres y mujeres aymara del norte de Chile, en las esferas social, económica, religiosa y sus representaciones, obtuvimos ciertos elementos significativos que permiten aproximarse a los modos por los que ellos construyen social y simbólicamente el género. Los datos etnográficos obtenidos mediante entrevistas, historias de vida y trabajo de campo, sugieren que estamos frente a un tema complejo, poco abordado en nuestro país, pero que nos da nuevas pistas de investigación en torno a las ideologías de género y sobre el estatus asignado a este en la cultura y sociedad aymara actual.

Partimos sosteniendo que el género es un principio organizativo fundamental de la vida de las comunidades aymara del altiplano y para demostrarlo se entregan antecedentes sobre las ideas de lo femenino y lo masculino, del ser mujer y ser hombre y sus vinculaciones con las prácticas sociales en las comunidades de Isluga y Cariquima, I Región de Tarapacá1. Así, indagar acerca de la posición de género en la organización social sugirió que para comprender las categorías sociales hombre-mujer y sus relaciones se deben tener en cuenta factores como la edad y los roles sociales. Asimismo, la mirada económica nos permitió una mejor aproximación a las dinámicas familiares donde el género y la generación fueron aspectos centrales. Finalmente, las exploraciones

\footnotetext{
* Resultado del proyecto FONDECYT No 1110980. Convenio de Desempeño Universidad de Tarapacá-MINEDUC.

** Universidad de Tarapacá, Departamento de Antropología, Arica, Chile. Correos electrónicos: amcarrasco@uta.cl; viviangav@yahoo.com
} 
en las ideologías de género demuestran que no solo son útiles para concebir las relaciones entre hombres y mujeres, sino también para pensar otro conjunto de relaciones de la organización social y del sistema religioso.

El documento comenzará con un breve recorrido sobre el estado de la cuestión respecto de los estudios de género en la región andina y el debate que el uso de este concepto siempre ha suscitado; luego se presentarán los resultados propiamente tales, que corresponde a los aspectos más relevantes que hemos podido concluir con el análisis de nuestros abundantes datos etnográficos; para finalizar con las consabidas reflexiones.

\section{Los Estudios de Género en la Región Andina}

Desde que comienzan a realizarse estudios de género en comunidades indígenas han surgido cuestionamientos respecto del uso de esta categoría de análisis para obtener un conocimiento más profundo e integral de las relaciones que se construyen entre mujeres y hombres, en un contexto cultural específico.

Particularmente, en el contexto de los pueblos aymara y quechua hablantes se ha argumentado que las reflexiones adolecen de seriedad científica, siendo más bien otro intento de colonización capitalista e imperialista. Entre las diversas críticas ha persistido aquella que cuestiona la aplicación de categorías occidentales en otros grupos culturales, otorgándoles no solo una determinada estructura y organización del mundo sino también significados que son particulares de nuestro propio mundo conceptual (Gavilán 1998:66).

Las opiniones en contra, generalmente problematizan la construcción del conocimiento occidental (Kothari 2001) y cuestionan la incorporación de visiones etnocéntricas para entender modos de vida y formular preguntas de investigación (Slater 2000). Con todo, también existen informes que trabajan la influencia de los estudios indios subalternos y postcoloniales en América del sur, señalando como paradoja que debates que surgen en el sur lleguen a países andinos intervenidos por el filtro académico del norte (Rivera y Barragán 1997).

En las comunidades andinas los estudios de género comienzan en la década de 1980, siendo un gran aporte para abrirse a esta nueva temática los estudios de la mujer, que surgen más tempranos. El debate gira, al inicio, en torno al tema de las relaciones de poder entre los géneros buscando su complementariedad o subordinación. Quienes planteaban o plantean la existencia de la complementariedad adhieren a la tesis de que la sociedad y cultura andina está estructurada en torno a una organización y pensamiento dual, cuyas relaciones determinan su especificidad étnica que asigna un estatus complementario tanto a hombres y a mujeres, así como a lo masculino y a lo femenino. En esta línea se argumenta que social o simbólicamente las diferencias de género no contienen desigualdades de poder marcadas (Cf. Isbell 1975; Harris 1985; Anderson 1990; Sánchez-Parga 1990). Por otro lado, la hipótesis de la subordinación fue promovida por los estudios sobre la situación de la mujer basada en las teorías feministas, postulando la existencia de dominación masculina (Cf. Bourque y Warren 1975; Casos 1990; De la Cadena 1991; Stolen 1987; Harvey 1989).

La mayoría de los estudios acerca de la sociedad y cultura andina no solo no han considerado este tema, sino que han evitado las categorías de lo femenino y lo masculino a pesar de su considerable presencia en la ideología religiosa (Cf. Albó 1992; Bastien 1996; Grebe 1981; Harris 1985; Martínez 1989; Montes 1986; Platt 1980 y 2003; Van Kessel 1992a, 1992b, 1994.

Se podría afirmar que la mayor parte de los estudios tienden a marcar la diferencia existente respecto de las sociedades nacionales para indicar que la posición y condición femenina en estas se presenta "más favorecida". Pero uno de los aspectos más problemáticos de la perspectiva de análisis que mayoritariamente se ha adoptado ha sido aplicar la lógica binaria del "andinismo": andino/europeo, capitalista/precapitalista, pagano/cristiano, tradicional/moderno. Y, en este contexto, se presupone una alteridad no contaminada al representar a la sociedad y cultura indígena como expresión de la continuidad de un pasado precolonial (Starn 1992; Bestard 2002). Así, se tiende a olvidar la permanente relación de intercambios culturales que se han producido en la historia y de la posición subordinada de las comunidades de origen indígena; se omite que nos situamos en un contexto de larga tradición colonialista en el que las personas no han sido entes pasivos, sino que sujetos activos de los procesos sociales y culturales (Gavilán 1996).

En esta línea se hallan los planteamientos de las dirigencias de las organizaciones aymara, para quienes el fundamento de la cosmovisión andina 
es la Pacha (el tiempo, la tierra, el mundo, espaciotiempo). En esta habitarían en forma armónica todos los elementos del universo, estructurados en forma dual, simbólicamente masculino y femenino, con cualidades opuestas pero formando una unidad en reciprocidad. Así, argumentan que una postura aymara de la relación hombre-mujer y en este sentido de género, refiere exclusivamente al Chacha-warmi (matrimonio, esposos, casados), término que se basa en la práctica ancestral de convivencia entre mujeres y hombres. Esta concepción tomaría en cuenta la complementariedad entre los géneros y no la igualdad, reconocería a hombres y a mujeres como diferentes, valorando esta diferencia porque los roles y funciones se caracterizan por su flexibilidad. Se sostiene, de esta manera, que las relaciones de género entre los aymara no acarrean desigualdades sociales entre mujeres y hombres y que sería el proceso de "transculturación" ocasionada por el "progreso, la modernidad y la migración campo-ciudad" la que ha hecho que la concepción de complementariedad del Chacha-warmi se vaya perdiendo. Con todo, este discurso concuerda con la tesis de la complementariedad entre los géneros (Ponencia de "Plan Andino" para el Encuentro Mujer y Desarrollo Rural, 1996).

Finalmente, centrándonos solo en los estudios realizados entre los aymara del Norte de Chile, advertimos que si bien existen aquellos que dan cuenta de la situación y condición de hombres y mujeres (Cf. Arriaza 1991; Carrasco 1993; Gundermann y González 2009; Pizarro 1988; Mamani 1999), estos no profundizan en la construcción simbólica del género. Solo a mediados de la década de los 90, y hasta nuestros días, comienzan a realizarse investigaciones que abordan el tema desde esta perspectiva (Cf. Gavilán 1996, 1998, 2001, 2002; Carrasco 1998, 1999, 2001, 2011; Carrasco y Gavilán 2009, 2011, 2012; Echeverría 1998a, 1998b, 2001), señalando la importancia que adquiere el conocimiento de las concepciones sobre género y sus diferencias en los procesos identitarios y obteniéndose interesantes referencias al papel fundamental que juega lo masculino y lo femenino como principio organizativo de la vida de este pueblo indígena.

Con todo y a pesar de las dificultades que se le puedan atribuir al uso del concepto de género, consideramos que como herramienta analítica y para los efectos de nuestro estudio, aporta una base teórica y conceptual útil para conocer las asociaciones simbólicas contenidas en las prácticas y en el discurso de la sociedad aymara. Concebir la diferencia entre mujeres y hombres entre lo masculino y lo femenino como una construcción histórica, social y cultural ha significado abrir la investigación en este ámbito de la realidad.

\section{Posición de Hombres y Mujeres en la Esfera Social, Económica y Religiosa}

Uno de los primeros resultados obtenidos al indagar en la esfera social del pueblo aymara es que la posición de hombres y mujeres se establece según un orden jerárquico en tres relaciones fundamentales. Siendo así, el estatus definido según la relación de parentesco (madre, padre, hijo, etc.) (ver Figura 1); el momento de su ciclo vital (Yocalla/ Imilla, Wayna/Tawajo, Chacha/Warmi, Achichi/ Apache) (ver Cuadro 1); y la relación fraternal, referida al lugar que se ocupe entre los/as hermanos/ as (menor, medio y mayor $)^{2}$. Lo anterior, mediante un proceso vivido por hombres y mujeres que los va situando en el lugar de mayor o menor prestigio. En otras palabras, podemos advertir que el género y la edad, según las fases estimadas por el pensamiento aymara, combinadas al rol asignado en la familia y la comunidad, son categorías necesarias para definir el carácter de las relaciones entre los individuos. De esta manera, podríamos establecer que según las categorías sociales de hombres y mujeres y de acuerdo al rol que estos desempeñen, el énfasis de la edad o el género en el carácter de las relaciones sería el siguiente:

Yocalla - Imilla $/$ hijo-hija $/$ hermano- hermana = edad Wayna - Tawajo / hijo-hija /hermano-hermana = edad Chacha - Warmi /esposo-esposa = género y rango social

Achichi - Apache /abuelo-abuela = género, edad y rango social

Ahora, en la relación con los parientes aliados adultos la edad sería más relevante que el género, considerando que los hombres como yernos (Tullqa) y las mujeres como nueras (Yuqch'a) serían nuevamente hijos e hijas.

La socialización aymara empieza durante la niñez a construir un patrón de diferencia de género, designándoseles con términos específicos y enfocándose progresivamente hacia aquellas conductas y habilidades apropiadas de los roles 


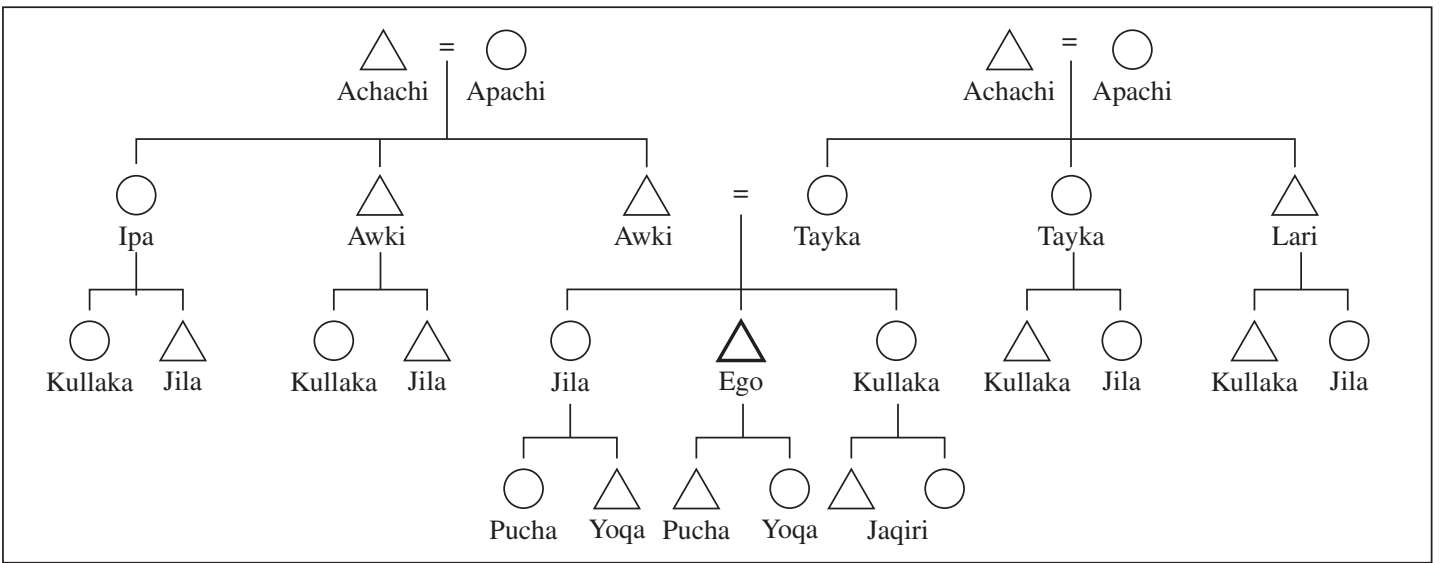

Figura 1. La terminología del parentesco.

Fuente: Carrasco y Gavilán, 2014.

Cuadro 1. Terminología aymara, ciclo vital y género

\begin{tabular}{|c|c|c|c|}
\hline \multirow{2}{*}{ Etapa } & \multicolumn{2}{|c|}{ Sexo } & \multirow{2}{*}{ Observaciones } \\
\hline & Hombre & Mujer & \\
\hline $\begin{array}{l}\text { Fetal } \\
\text { Desde la gestación hasta antes de nacer. }\end{array}$ & \multicolumn{2}{|c|}{ Sullu } & Sin distinción de género. \\
\hline Primeros seis meses (a veces hasta menos meses). & \multicolumn{2}{|c|}{ Asu wawa } & Sin distinción de género. \\
\hline $\begin{array}{l}\text { Entre los seis meses hasta cuando baja de la } \\
\text { espalda de la madre y comienza a andar. }\end{array}$ & \multicolumn{2}{|c|}{ Wawa } & Sin distinción de género. \\
\hline $\begin{array}{l}\text { Desde que comienza a caminar hasta los } 3 \\
\text { o } 4 \text { años }\end{array}$ & Wawa Yuqalla & Wawa Imilla & $\begin{array}{l}\text { Sigue siendo considerado Wawa, pero se empieza } \\
\text { a distinguir su sexo }\end{array}$ \\
\hline $\begin{array}{l}\text { Entre los } 4 \text { y } 14 \text { años (las edades límites } \\
\text { pueden variar). }\end{array}$ & $\begin{array}{l}\text { Jisk'a } \\
\text { Yuqalla } \\
\text { Jach'a }\end{array}$ & $\begin{array}{l}\text { Jisk'a } \\
\text { Imilla } \\
\text { Jach'a }\end{array}$ & $\begin{array}{l}\text { Distinción de género. } \\
\text { Al término se les pueden agregar otras } \\
\text { denominaciones: } \\
\text { Jisk'a (chico o menor) si son más pequeños, o } \\
\text { Jach'a (grande) si son más mayores. }\end{array}$ \\
\hline $\begin{array}{l}\text { Preadolescencia, entre los } 11 \text { a } 14 \text { años } \\
\text { (solo en algunos sectores altiplánicos). }\end{array}$ & Majta & Maldaya & $\begin{array}{l}\text { Distinción de género. } \\
\text { Correspondería también a las denominaciones } \\
\text { Jach'a Yoqalla y Jach'a Imilla, respectivamente. }\end{array}$ \\
\hline $\begin{array}{l}\text { Adolescencia, aproximadamente después de los } \\
14 \text { años hasta el momento de casarse. }\end{array}$ & Wayna & Tawaqu & Distinción de género. \\
\hline Adulto. Después de casarse. & Chacha & Warmi & Distinción de género. \\
\hline Adulta, aún en etapa reproductiva. & - & $\begin{array}{c}\text { Tayka } \\
\text { (o mama) }\end{array}$ & $\begin{array}{l}\text { Distinción de género. } \\
\text { También usado como término de parentesco que } \\
\text { designan a padre (Auki) y madre. }\end{array}$ \\
\hline $\begin{array}{l}\text { Mayores de edad. Sobre } 50 \text { años. } \\
\text { Mujer no reproductiva. }\end{array}$ & $\begin{array}{l}\text { Jach'a awki } \\
\text { (o Jach'a } \\
\text { tata) } \\
\text { Achachi }\end{array}$ & $\begin{array}{l}\text { Jach'a tayka } \\
\text { (o Jach'a } \\
\text { mama) } \\
\text { Apachi }\end{array}$ & $\begin{array}{l}\text { Distinción de género. } \\
\text { Se agrega el término Jach'a para designar que } \\
\text { son "mayores" o "grandes". } \\
\text { Denominaciones de abuelo/a, dentro del sistema } \\
\text { de parentesco. }\end{array}$ \\
\hline Individuo después de la muerte. & & & $\begin{array}{l}\text { Sin distinción de género. } \\
\text { Concepto más general que alude a todos los } \\
\text { muertos. }\end{array}$ \\
\hline
\end{tabular}

Fuente: Carrasco, A. M. 2014. 
de cada sexo, iniciándose la identificación hacia el mundo del trabajo especificado por género. En la adolescencia se marca esta distinción entre lo que debe ser un hombre y una mujer, situación que en la adultez adquiere dominios claramente diferenciados. Lo enseñado familiarmente corresponde a un adiestramiento para la vida adulta, se va educando en los caminos social y culturalmente definidos de cada género y es en la madurez y con el matrimonio (Chacha-warmi) cuando culmina el aprendizaje, donde se ejecuta plenamente la división del trabajo por género y se exhiben los comportamientos esperados. Así, desde el punto de vista de los actores sociales, el tránsito de una fase a otra es conseguido por un camino igualitario; sin embargo, se podría argumentar que si bien la posición de hombres y mujeres en las fases previas al matrimonio no difiere sustantivamente, cambia con este. La relación Chacha-warmi es jerárquica, ya que el esposo se sitúa en la posición de mayor prestigio y la esposa en la de menor prestigio; estas posiciones de género desiguales entre mujeres y hombres se observan con mayor claridad en y a partir de la división sexual del trabajo. Ahora, hay que considerar que en tanto madres, suegras o abuelas se sitúan en el lugar más valorado y de más poder, respecto de hijas, nueras y nietas, pero frente a sus "pares" masculinos estas siguen en segundo plano.

El rol político y económico asignado a la unidad Chacha-warmi nos señala que la relación en ella contenida dquiere mayor relevancia en la vida de las personas. El carácter exogámico y virilocal de la residencia matrimonial y la adscripción de los hijos a la familia del varón sitúa a la mujer en una posición de mayor ambigüedad que la de los hombres. A nivel de su propio grupo doméstico es considerada un miembro temporal, ya que dejará de pertenecer a él para adscribirse a otro: el de su marido. Su filiación será en la práctica transitoria, por tanto se considerará una inversión para terceros, siendo el producto de su trabajo más personal que familiar; sin embargo, esta condición es esencial para la relaciones de intercambio que supone la alianza con otras unidades domésticas.

La dinámica de cambios que actualmente se van dando en las relaciones interfamiliares, especialmente en el ámbito del trabajo, aparentemente han disminuido la importancia que tiene esta como motor de las redes de intercambio. Esto puede percibirse también en el hecho de que las ceremonias tradicionales de casamiento (Kasarata) aún expresan la subordinación del varón y su familia en la petición de la novia, siendo la mujer y su familia las que asumen la posición de poder, ya que aceptan o rechazan al futuro marido. Pero en el casamiento, las exigencias que se plantean a la mujer en la incorporación a la unidad doméstica del varón y las exigencias impuestas al yerno para ser considerado parte integrante de la familia de la mujer son prácticas cada vez menos consideradas. Los términos que usa la esposa para referirse a sus nuevos parientes de la familia del marido son los mismos que usa el marido, es decir, padre, madre, hermano, etc.; los que usa el marido para referirse a los de ella (esposa) son los de los aliados, o sea, suegro, suegra, cuñado, etc. Sin embargo, en aymara estos son los mismos por el lado de la mujer y del hombre (ver Figura 2).

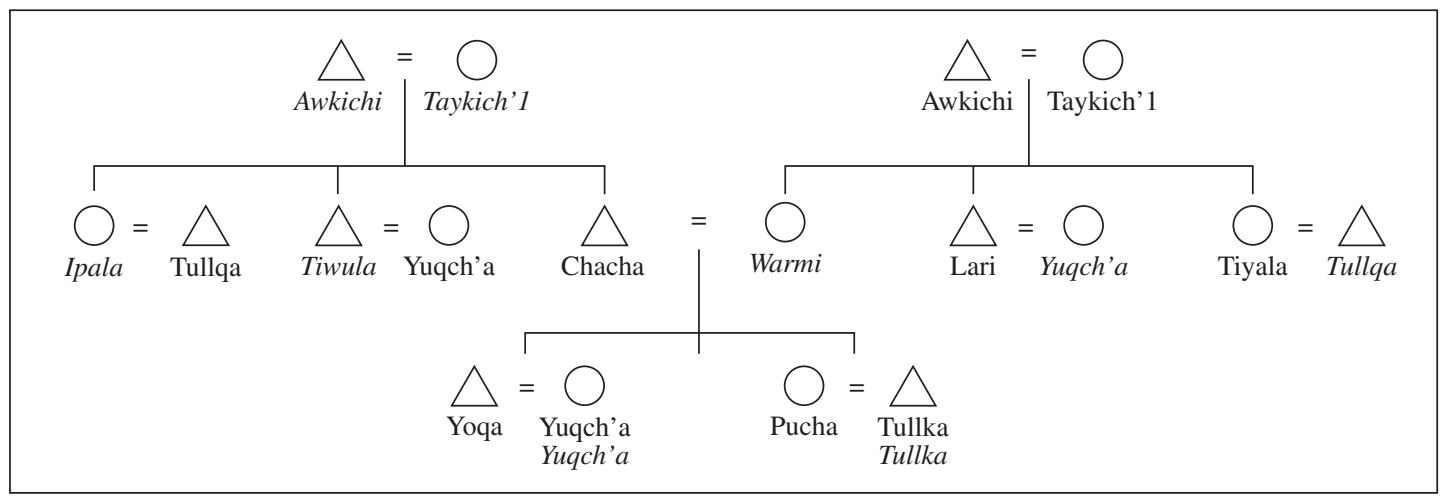

Figura 2. Terminología aymara, ciclo vital y género.

Término desde la Mujer.

Término desde el Hombre.

Fuente: Carrasco y Gavilán, 2014. 
La importancia de la alianza en la posición y estatus de las personas es algo que debe ser profundizado, pues existen casos de casamientos entre cuñados que pueden contener elementos para comprender mejor las relaciones entre los grupos que vincula el matrimonio. También, encontramos cierta tendencia a practicar el levirato y sororato, especialmente presente en generaciones mayores (abuelos y bisabuelos), que podrían dar cuenta de la existencia de preferencias matrimoniales. Por otra parte, no son pocos los testimonios de hombres y mujeres que indican la vigencia y relevancia de la capacidad de decisión de las madres y los padres en la elección del esposo o esposa, aun cuando hay que dejar claro que la tendencia mayoritaria actual es que sean los propios involucrados los que decidan. Es este sentido, podemos hablar de hombres y mujeres célibes, siendo tanto objetos como sujeto de intercambio.

Lo anterior permite pensar en la importancia que pueden tener las relaciones de parentesco y matrimonio para entender la posición de las mujeres (Harvey, 1989). Tanto el lugar que ocupa en las relaciones consanguíneas como en las de alianza se entienden en términos jerárquicos; así también, la ambigüedad de su posición no solo ocurriría en su condición de esposa sino también se extendería a su grupo doméstico original. Sus capacidades reproductivas y su inserción en la familia del marido hacen que la mujer se halle en un lugar conflictivo, pero también lo es su posición en las relaciones consanguíneas debido a la concepción de la propiedad individual (ganado y bienes adquiridos con trabajo), al carácter virilocal de la residencia matrimonial y a la adscripción de la descendencia a la familia del hombre.

Visto lo anterior, se podría argumentar que la valoración social o prestigio se obtiene con la combinación de mecanismos adscritos (género, edad, roles) y adquiridos (trayectoria social: matrimonio, cargos sociales y políticos, riqueza, etc.). En este sentido, el género es uno más de los componentes de las estructuras de prestigio y por tanto el resultado final del estatus de las personas se definirá de acuerdo con una combinación de ellos. Así, podemos colegir que las categorías hombre y mujer no son homogéneas y en consecuencia sus relaciones serán también variables.

La salida de la mujer de su grupo doméstico implica la exclusión de la propiedad de la tierra y también de las herramientas de trabajo agrícolas.
Lo que las hace más dependientes de los varones; no obstante, en la práctica los propietarios son los adultos mayores, de quienes dependen tanto hombres como mujeres, especialmente los recién casados. Ambos tienen derecho a uso por su sola condición de pertenencia o adscripción al grupo doméstico, lo que es válido también para las mujeres "abandonadas" por los maridos, quienes tienen derecho a quedarse en la comunidad. Así, serán los varones los poseedores, lo que hace más vulnerables a la posición femenina.

El enérgico énfasis existente en la posesión individual de los bienes, permite la posesión individual del ganado, aspecto central en la economía familiar altiplánica, y cierto control sobre su trabajo y productos, aspectos que relativizan el control ejercido por los varones sobre las mujeres. Por otra parte, hay que mencionar que hoy se dan intensos procesos de movilidad de la población aymara rural a pueblos del desierto y ciudades costeras (Carrasco y González 2012); pero pese a los cambios que esto provoca, fundamentalmente en el hecho de que la tierra y su propiedad deja de ser relevante, la división sexual del trabajo se transforma y las mujeres que quedan en el sector rural asumen participación en reuniones de la comunidad, las posiciones de mujeres y hombres no varían, por lo que podríamos hipotetizar que el control de los recursos productivos y la participación social no serían las únicas fuentes de explicación para la posición de género (Sacks 1979; Leacock 1991).

La habitual división sexual del trabajo, de la unidad familiar campesina, sí expone diferencias de género, como ya lo mencionáramos. No tanto en el plano de la producción, como en el de la reproducción, observándose mayores privilegios para los hombres y recargo de trabajo para las mujeres. En términos políticos, la posesión de la tierra otorga a estos últimos mayores capacidades de decisión y legitima su orientación hacia las actividades de representación. Mientras las mujeres junto con participar en las tareas productivas es la principal encargada de las reproductivas. Esta situación de aumento de trabajo para las mujeres podría ser producto de cambios en las actividades de ambos géneros que hace ya un par de décadas se vienen dando, pues observamos la tendencia a incorporar los roles de mujeres y hombres no aymaras urbanos. Los hombres consideran como labores domésticas femeninas el hilado, el tejido y el lavado de ropa. Aunque un motivo fundamental son los mayores 
niveles de mercantilización de la economía altiplánica y la presencia del Estado, especialmente por medio de la escuela nacional (Carrasco y Gavilán 2012). Los varones adultos se desligan del trabajo de pastoreo y cuidado del ganado para dedicarse más al comercio y otras actividades remuneradas en la zona o en pueblos y ciudades; además, quienes remplazan la ausencia de los hijos/as en edad escolar en las tareas domésticas son las madres. Podemos inferir, entonces, que la división sexual del trabajo es, entre los aymara, una fuente de diferenciación social (Bourque y Warren 1976; De la Cadena 1991; Gavilán 2002).

Si seguimos analizando las actividades, productivas y reproductivas ${ }^{3}$, que realizan hombres y mujeres aymaras, no cabe duda que se expone claramente una sociedad en transformaciones, donde la categoría Wayna (hombre joven, soltero) aparece en cierto modo desvinculada de la producción y reproducción de las familias campesinas. Las mayores oportunidades de educación que estos reciben en relación con las jóvenes, ha sido explicada por los/as entrevistados/as, debido a la necesidad que, históricamente, han tenido los hombres de ser los representantes de la familia ante la comunidad y el Estado. Mientras que en las mujeres la educación es considerada una mala "inversión", ya que con su casamiento se incorporarán a otra unidad doméstica y, además, no tienen la obligación de representación, especialmente ante la sociedad nacional. Pero está claro que en la práctica la educación supone mayores capacidades de información, manejo de las relaciones extracomunidad y mayor prestigio para quienes poseen niveles avanzados; por tanto, la condición de las mujeres va detrás de los varones. Con todo, hay que decir que esta situación ha cambiando enormemente, constatándose cada vez más un aumento en los niveles de escolaridad de las jóvenes (Carrasco y Gavilán 2012; Echeverría 1998c).

En el plano sociopolítico las mujeres casadas, Warmi, no son del todo excluidas, ellas en su condición de esposas tienen el derecho y el deber de participar en las actividades sociales comunales, junto a su marido, pero esta se ubica en un segundo plano. Situación que se agudiza por las exigencias que imponen las relaciones entre las comunidades con el Estado y el mercado, ya que serán los varones quienes las dominen mejor.

Aun cuando seamos conscientes de los grandes cambios que ha vivido la sociedad aymara y de los avances y logros de las mujeres en lo que a una mayor participación social se refiere, es claro que ellas no tienen la misma presencia que los hombres en el espacio público. Esto es cierto, ya que están más vinculadas y presentes en lo doméstico, pero más que un control de parte de los hombres por mantenerlas en este espacio es el Estado quien ha tenido un rol central. Se percibe cierta tendencia a la diferenciación, por parte de los comuneros, entre las dinámicas públicas internas (propias y comunitarias) y externas (vinculación con sociedad mayor). En este sentido, si se considera que el espacio público se halla en el ámbito de las relaciones externas, entendidas como acciones impuestas desde el Estado y vinculaciones con la sociedad mayor, es claro que las mujeres se encuentran en desventaja; pero si este se halla en la participación comunitaria (asamblea comunal, cargos sociales, relaciones entre las unidades domésticas, etc.), la participación femenina aumenta, debido a que estas pueden asumir un conjunto de tareas y responsabilidad de representación igual que los hombres en su condición de esposas y madres, más aún y como ya dijimos, en casos de migración temporal de los hombres del hogar. Sin embargo, la vida social actual transcurre en una combinación de ambos espacios, donde lo externo se impone cada vez más; por lo que el resultado final del análisis de las relaciones de poder asigna mayores privilegios a los hombres.

Por otra parte, si bien tanto hombres como mujeres son considerados socialmente adultos con el matrimonio, las relaciones conyugales asignan mayor poder a los primeros. Para entender esto hay que partir diciendo que las razones principales de conflictos de pareja se dan en torno al trabajo, concebido según los estereotipos masculino o femenino y donde las mujeres/esposas asumen el rol de proveedoras y son las encargadas de la reproducción biológica, de la mano de obra y de la unidad social (Gavilán 2002:115), y la infidelidad femenina. En este sentido, observamos dos aspectos centrales. Primero que entre las partes en desacuerdo debe haber mecanismo de mediación: padrinos y padres de los contrayentes y resquicios morales y legales de solución. Segundo, que las mujeres son consideradas potencialmente infieles y ello es sancionado. Esta última cuestión nos lleva a conjeturar que la sexualidad femenina y sus capacidades reproductivas son una condición particular que debe ser controlada por mecanismos sociales y morales, pero igualmente pueden 
transgredirse. La fertilidad, la sangre y la reproducción biológica es dotada de fuerza y poder y son elementos asociados a lo femenino (Carrasco y Gavilán 2005, 2009; Platt 2003).

Pero pese a lo anterior, nuestros avances investigativos nos indican que en el espacio de la sexualidad, el hombre y la mujer, lo femenino y lo masculino no se estructurarían en una relación de jerarquía. La simbolización de los cuerpos de hombres y mujeres y de la reproducción humana entre los aymara nos hace pensar que la dominación masculina puede ser explicada por el prestigio que se le otorga a los componentes del cuerpo humano vinculados a la reproducción sexual. Pero las categorías de la diferencia sexual son complejas; entre los aymaras esta diferencia sexual releva una diversidad de aspectos de los cuerpos humanos y no tan solo el aparato genital como sucede en la cultura "occidental". Por otra parte, el hecho que es la sexualidad heterosexual la que produce, la que es biológicamente fértil (las relaciones homosexuales son posible, ocurren incluso entre seres divinos, pero no produce frutos), hace que tanto el cuerpo femenino como el masculino sean de importancia central: se sitúan en planos diferenciados pero iguales. En este sentido, las fuentes de prestigio y poder no se ubicarían en este nivel; es decir, la posición que ocupa cada sexo en la reproducción no explicaría las relaciones de poder existentes entre ambos.

Así, la información obtenida nos muestra que el cuerpo humano remite a una esencia "biológica". Sobre la base de esta, las ideas acerca del cuerpo, sexo y sexualidad femenina y masculina se construyen las representaciones de cada género. Pero entre los aymara parece ser el carácter esencialista de las diferencias de los cuerpos de mujeres y hombres el que limita las posibilidades de transformación en las relaciones de poder entre los géneros. A pesar de asignar una mayor complejidad a lo femenino en la reproducción sexual y social de la comunidad, se construyen relaciones de poder a partir de la valoración dada a sus componentes en términos de prestigio, y bajo estos términos se dan las desigualdades sociales, como la violencia en contra de las mujeres o su exclusión de los principales medios de producción (Carrasco y Gavilán 2009).

Haciendo referencia a la organización social y espacial de la comunidad, podemos decir que esta también es pensada simbólicamente en términos de género. Entre cada una de las mitades (Arajj Saya y Manqha Saya, organización espacial y social de la comunidad) se construye una diferenciación jerárquica, donde Arajj Saya o mitad de arriba es masculina, por lo que ocupará la posición de mayor prestigio respecto de Manka Saya o mitad de abajo, concebida como femenina, la que ocupa el segundo lugar en prestigio y estatus; observamos, entonces, que la oposición arriba/abajo es pensada de manera diferenciada en su valoración. Estas contienen implícitamente referencias sexuales y en relación asimétrica (mayor o menor prestigio y poder) y se concebirían como una ampliación a mayor escala del modelo provisto por la pareja humana, la que estaría constituida por dos individuos distintos y diametralmente opuestos en su polaridad sexual y en sus atributos. Esta oposición se resolvería con la cópula, comunión entre macho y hembra. Así, las parcialidades concebidas simbólicamente en términos de género se unen en un acto sexual también simbólico: el Tinku, combate ritual entre la mitad masculina y la femenina.

También podemos dar cuenta que el lugar que ocupan hombres y mujeres como sus representaciones, en las ceremonias religiosas, permite identificar una segunda oposición en las clasificaciones de género que sería derecha/izquierda, donde la primera corresponde a lo masculino y de mayor prestigio respecto de la segunda, femenina $\mathrm{y}$ de menor prestigio.

Hemos visto que las diferencias de género sirven tanto para establecer la posición social y prestigio de hombres y mujeres, pero también para organizar el mundo religioso. Uno de los aspectos más resaltantes en los datos sobre religiosidad obtenidos es la persistencia del uso de las categorías femenino-masculino como principio para establecer las relaciones entre los distintos sujetos que componen la cosmovisión aymara. Los contenidos asignados a estas categorías, en un juego permanente de analogías, puede hacer comprensible el funcionamiento de la vida en el mundo y en la comunidad.

El análisis de las divinidades y sus relaciones dan cuenta de jerarquías no solo entre entidades de un mismo sexo-género, sino especialmente entre masculinos y femeninos. Dios Inti y Pacha Mama son los que lideran el panteón, siendo lo masculino el término con mayor prestigio. Siempre se le nombra a él primero, y su posición geográfica y ritual ocupa el lugar más preciado: arriba y a la derecha (ver Cuadro 2). Lo mismo 
Cuadro 2. Las ideas de la diferencia en la religiosidad aymara

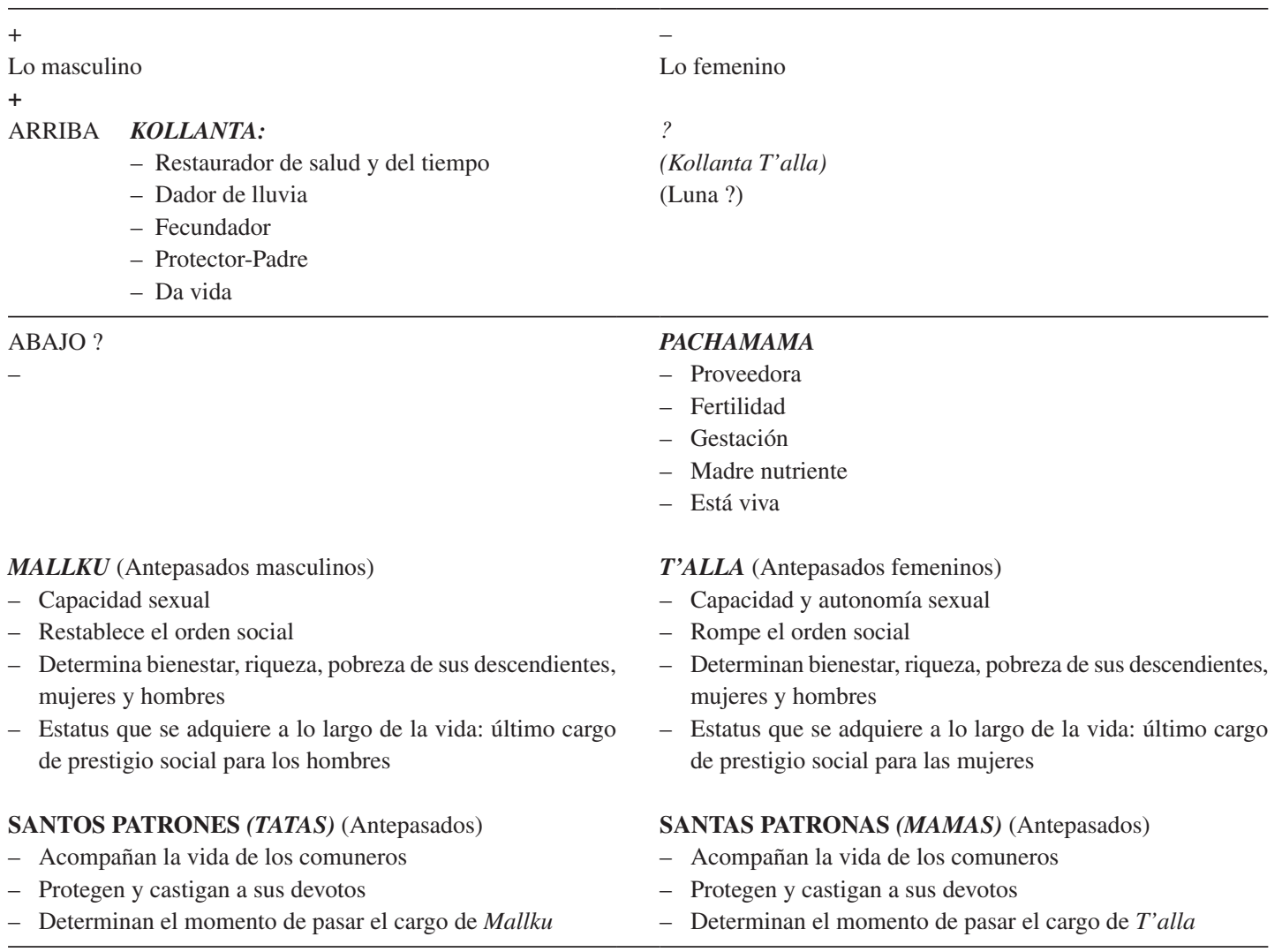

Fuente: Gavilán, V. 2014.

ocurría con los caciques de la Sayas (mitades) o los Ayllus (agrupación de individuos o familias descendientes de un antepasado común) comunales: el de arriba, asociado a Saya masculina con mayor prestigio que la de abajo, vinculado a lo femenino. A pesar de estas posiciones, parece no haber una clara oposición en lo masculino y lo femenino. Tanto seres masculinos como femeninos ejercen poder sobre otros de menor jerarquía de género homólogo o no. El hecho de que existe la posibilidad que una deidad sea hombre y mujer al mismo tiempo, que los Uywiris cerros pueden comer y violar a mujeres y a hombres relativiza las oposiciones. Esto, asociado a la ambivalencia de las deidades: milagroso/peligroso, al bien y al mal, nos habla de una relación más compleja que la simple oposición existente en la tradición judeocristiana. Es decir, el rol que cumplen y la importancia asignada a las divinidades es tan o más importante que su género (Cf. Gavilán 1998).

\section{Conclusiones}

Las indagaciones sobre lo masculino y lo femenino, acerca de las ideas de ser mujer y ser hombre, en las distintas esferas de la vida social y religiosa de los aymaras, nos señalan un rico e interesante ámbito de investigación que, sin dudas, es necesario profundizar.

Hemos visto que una de las cualidades que diferencia a las mujeres de los hombres y a lo femenino de lo masculino es el prestigio; lo que se expresa en la existencia de jerarquías, donde a lo masculino se le asigna mayor grado de prestigio, otorgándoseles mayores grados de autoridad y poder. Sin embargo, la categoría de género se cruza con otros sistemas de prestigio: la edad o fase del ciclo vital y el rango social que otorga el "sistema de cargos comunales". Ello hace que lo femenino y la mujer puedan situarse también en rangos superiores respecto de seres masculinos. 
Ahora, a partir de la información expuesta es posible pensar que cuando lo femenino se superpone a lo masculino, exceptuando los casos en los que la edad o rango social son más determinantes, los elementos que marcan la diferencia refieren a la sexualidad, la fertilidad y la maternidad; características asociadas también a la abundancia económica, suerte y bienestar familiar. Tal vez estas ideas son las que abren la posibilidad a las mujeres y a lo femenino a transgredir el orden social establecido. Mientras a ellas se les otorga dotes de transgresoras, a los hombres se les piensa como restablecedores, mediante un ejercicio de poder que no tienen las primeras, como la violencia en contra de ellas o de otros hombres. La patrilinealidad y patrilocalidad le otorga a los hombres cuotas de poder que las mujeres no tienen, estableciéndose la posición de hombres y mujeres según un orden jerárquico.

La información sugiere que el género, la edad y los roles se organizan a base de un principio común y este es el de las jerarquías. Las relaciones sociales se conciben en términos de posicionamientos diferenciados, donde una de las partes posee mayor o menor prestigio y menor estatus respecto de la otra. Pero una de las características de las relaciones jerárquicas es que son dinámicas y pueden ser transitorias. Otra característica destacable es que la relación entre dos o más partes en posiciones diferentes, con poderes diferenciados, requieren de una mediación: un tercer elemento que se constituya como mediador para la resolución de los conflictos. Esta mediación supondría restablecer el orden, volver a un estado inicial jerárquico.

A partir de lo expuesto, creemos que el género adquiere tanta relevancia en la diferenciación social como la edad y los roles. Este es concebido, al igual que otras clasificaciones, dentro de un orden jerárquico. La construcción social y simbólica del género se estructura, entonces, en torno a jerarquías que se definen por su asociación a la fase del ciclo vital y al rol social, los que conforman el contexto que determinará la posición y condición de hombres y mujeres y la valorización de lo femenino y lo masculino. Pero cuando se establece entre "iguales", igual edad e igual rol social la mujer y lo femenino ocupa el segundo lugar; esto significaría que las diferencias de género sitúan al hombre y lo masculino en la posición de mayor prestigio.

El carácter "situacional" y transitorio del estatus y prestigio de las personas puede ser el motivo por el que el discurso aymara insista en la igualdad social entre el hombre y la mujer; es decir, resulta dificultoso considerar solo las condiciones de género sin su contexto específico. Pero desde el punto de vista de las ideologías de género el menor o mayor prestigio no implicaría desigualdad social, sino simplemente diferencia. Lo que ocurriría también con los otros componentes como la edad, los roles o la posición de clase.

De acuerdo con lo anterior, las posturas que adhieren a que la construcción simbólica del género en las comunidades andinas se estructura en torno a un sistema de pensamiento dual basado en la oposición masculino/femenino como términos complementarios e igualitarios no se sostienen. Podemos ver que uno de los componentes centrales en las relaciones sociales es la diferenciación del estatus y prestigio que define la posición de las personas y esta puede basarse en el género, edad y roles; como también, en las trayectorias personales y, esta diferenciación, construye desigualdades sociales que provocan diferencias en el ejercicio del poder.

\section{Agradecimientos}

La información primaria se obtuvo por medio de entrevistas y observación participante, realizadas durante largas temporadas de campo (durante tres años) y permanencias cortas en los últimos años. Igualmente se ocupó material etnográfico disponible en el Taller de Estudios Andinos, al que se le agradece.

\section{Referencias Citadas}

\footnotetext{
Arriaza, $\mathrm{P}$.

1991 Fuentes Bibliográficas para el Estudio de los Aymaras del norte de Chile. El Jote Errante, Iquique. Albó, X.

1992 La experiencia religiosa aymara. En Rostros Indios de Dios, coordinador M. Marzal, pp. 326-353, Hisbol, La Paz.
}

Anderson, J.

1990 Sistema de Género e Identidad de Mujeres en Culturas Marcadas del Perú. Revista Peruana de Ciencias Sociales, Vol. 2, 1:37-68, Lima, Perú.

Bastien, J.

1996 [1978]. La Montaña del Cóndor. Metáfora y Ritual en un Ayllu Andino. Hisbol, La Paz. 
Bertonio, L.

[1612] 1956. Vocabulario de la lengua aymara. Universidad Mayor de San Andrés, La Paz.

Bestard, J.

2002 Identidades, relaciones y contextos. En Estudis d'antropologia Social i Cultural, coordinador J.B. Camps, Departament d'Antropologia Cultural I Historia d'America I Africa. $N^{\circ} 7$, Universitat Barcelona, España.

Bourque, S. y Warren K.B.

1982 "Campesinas y comuneras: subordinación en la sierra". Mujer Andina $\mathrm{N}^{\circ} 1$, Puno.

Casos, V.

1990 La Mujer Campesina en la Familia y la Comunidad. Ediciones Flora Tristán, Perú.

Carrasco, A.M.

1994 Mujer aymara y trabajo remunerado. Revista Temas Regionales 1:30-41. Arica, Chile.

Carrasco, A.M.

1998a Mujeres aymara e inserción laboral. Revista de Ciencias Sociales, 8: 83-96, Ediciones Universidad Arturo Prat, Iquique.

Carrasco, A.M.

1998b. Constitución de género y ciclo vital entre los aymaras contemporáneos del Norte de Chile. Chungara Revista de Antropología Chilena 30: 87-103, Arica.

Carrasco, A.M.

2001 Violencia conyugal entre los aymaras del altiplano chileno: Antecedentes para comprender las relaciones de género en el matrimonio. Revista de Ciencias Sociales, 2: 85-96, Ediciones Universidad José Santos Ossa, Antofagasta.

Carrasco, A.M.

2011 "Paradigmas Éticos y Morales en la Construcción de la Sexualidad de Hombres y Mujeres Indígenas y no Indígenas en el norte de Chile". Revista Nuevas Tendencias en Antropología (NTA), 1:1-19, Universitas Miguel Hernández, España.

Carrasco, A.M. y V. Gavilán

2005 Sexualidad y género: La unidad de lo femenino y lo masculino como símbolos de reproducción y fertilidad entre los Aymara del norte de Chile. En Imaginarios, Identidades e Historias. Miradas desde la Antropología del Género, compilado por L. Rebolledo et al., pp. 169-186, CEC, Universidad Autónoma de Baja California.

Carrasco, A.M. y V. Gavilán

2012 Influencia del proceso de enseñanza escolar fiscal en la socialización de mujeres y hombres aymara de la zona altiplánica del norte de Chile. Revista Estudios Atacameños 44: 73-88, Antofagasta.

Carrasco, A.M. y V. Gavilán

2009 Representaciones del cuerpo, sexo y género entre los aymara del norte de Chile. Chungara Revista de Antropología Chilena 41: 83-100. Arica.

Carrasco, A.M. y González, H.

2012 La movilidad poblacional aymara en tiempos de postcomunalidad. En Actas XXXIV Convegno Internazionale di Americanistica, Perugia, Italia.

De la Cadena, $\mathrm{M}$.

1991 Las Mujeres son más Indias. Etnicidad y Género en una Comunidad de Cusco. Revista Andina, Centro "Bartolomé de Las Casas", año 9, 1: 7-47, Perú.

Echeverría, C.

1998a De Hombre a Persona, de Mujer a Esposa: una aproximación a la división del trabajo por género dentro del matrimonio en Isluga y Cariquima. En Actas del $3^{\circ}$
Congreso Chileno de Antropología Desafíos para el tercer milenio, Santiago, Chile.

Echeverría, C.

1998b División sexual del trabajo y matrimonio aymara contemporáneo desde una perspectiva de género. Revista de Ciencias Sociales 8: 97-108, Universidad Arturo Prat, Iquique.

Echeverría, C.

1998c Estado y Educación Rural en el Norte de Chile. Ediciones Taller de Estudios Andinos, Serie Documentos de Trabajo, Arica.

Echeverría, C.

2001 Un viaje a la visibilidad: las investigaciones sobre los aymaras de la Región de Tarapacá y la Relación Género-Etnicidad como parte de un Debate Pendiente. En Actas del $5^{\circ}$ Congreso Internacional Género y Realidad Andina, Antofagasta.

Gavilán, V.

1996 Mujeres y Hombres en Isluga y Cariquima: Una Aproximación a las Relaciones de Género Entre los Aymarás del Norte de Chile. Tesis para optar al grado de Magíster en Antropología, FLACSO, Quito.

Gavilán, V.

1998 Elaboraciones de género en la religiosidad de mujeres y hombres Aymara del norte de Chile avances de investigación. Revista de Ciencias Sociales 8: 65-82, Ediciones Universidad Arturo Prat, Iquique.

Gavilán, V.

2001 Los Rituales Propiciatorios de la vida: un Ensayo de Comprensión de las formas Simbólicas del Género en las Comunidades Aymaras del norte de Chile. Ediciones Taller de Estudios Andinos, Serie Documentos de Trabajo, Arica.

Gavilán, V.

2002 'Buscando Vida': Hacia una Teoría Aymara de la División del Trabajo por Género. Chungara Revista de Antropología Chilena 34: 101-117.

Grebe, $\mathrm{M}$.

1981 Cosmovisión Aymara. Revista de Santiago 1: 61-79.

Gundermann, H. y H. González.

2009 Sociedades Indígenas y Conocimiento Antropológico. Aymaras y Atacameños de los Siglos XIX y XX. Chungara, Revista de Antropología Chilena 41: 113-164. Arica.

Harvey, P.

1989 Género, Autoridad y Competencia. Lingüística, Participación Política de la Mujer en Pueblos Andinos. I. E.P., Documento de trabajo, No 33, Lima, Perú.

Harris, O.

1985 Una Visión Andina del Hombre y la Mujer. Revista Allpanchis, Vol. XXI, pp. 25: 17-39, Cusco-Perú.

Isbell, B.J.

1975 La Otra Mitad Esencial, Un Estudio de Complementariedad Sexual en los Andes. Revista Estudios Andinos, año V, 1: 37-56, Perú.

Kothari, U.

2001 Feminist and Postcolonial Challenges to Develoment. En Kothari, U. y M. Minogue (eds.). Development Theory and Practice Critical Perspectives. Pagrave: Basingtoneke.

Leacock, E.

1991 La Interpretación de los Orígenes de la Desigualdad entre los Géneros: Problemas Conceptuales e Históricos. En El Género en Perspectiva: De la Dominación Universal a la Representación Múltiple, Universidad Metropolitana, México. 
Mamani, M.

1999 Chacha-Warmi Paradigma e Identidad Matrimonial Aymara en la Provincia de Parinacota. Chungara, Revista de Antropología Chilena 31: 307-317, Arica.

Mamani, M.

2002 Diccionario Práctico Bilingüe Aymara - Castellano. Zona Norte de Chile. Emelnor Norprint, Antofagasta, Chile.

Martínez, G.

1989 Espacio y Pensamiento en los Andes Meridionales. Hisbol, La Paz.

Montes, F.

1986 La Máscara de Piedra. Simbolismo y Personalidad Aymaras en la Historia. Editorial Quipus, La Paz.

Pizarro, L.

1988 Algunas Reflexiones Acerca de los Espacios Femeninos y Masculinos en las Comunidades Aymara del Sector de Isluga. Documento de Trabajo, $\mathrm{N}^{\mathrm{o}} 10$, TER, Iquique, Chile.

"Plan Andino"

1996 Encuentro Mujer y Desarrollo Rural, Arica.

Platt, T.

1980 Espejos y maíz: El concepto de yanantin entre los macha de Bolivia, en: Mayer, E. y Bolton, R. (eds.), Parentesco y matrimonio en los Andes, pp. 139-182, Pontificia Universidad Católica del Perú, Lima.

Platt, T.

2003 El feto agresivo. Parto, formación de la persona y mito-historia en los Andes. Revista Estudios Atacameños 22:127-155, San Pedro de Atacama, Chile.
Rivera, S. y R. Barragán

1997 (eds.). Debates Post Coloniales: Una Introducción a los Estudios de la Subalternidad. SEPHID-Aruwiyiri, La Paz-Rotterdam.

Sánchez-Parga, J.

1990 ¿Por qué Golpearla? Ética, Estética y Ritual en los Andes. CAAP, Centro Andino de Acción Popular, Ecuador.

Sacks, K.

1979 Engels Revisitado: las Mujeres, la Organización de la Producción y la Propiedad Privada. En Antropología y Feminismo, editores K. Young, y O. Harris, Anagrama, Barcelona, España.

Starn, O.

1992 Antropología andina, "Andinismo y Sendero Luminoso". Revista Allpanchis 39: 73-92, Perú.

Slater, D.

2000 The process and prospect of political geography. Revista Political Geography 19(1): 1-3.

Stolen, K.A.

1987 A Media Voz. Ser Mujer Campesina en la Sierra Ecuatoriana, CEPLAES, Quito, Ecuador.

Van Kessel, J.

$1992^{a}$ Holocausto al Progreso. Los Aymaras de Tarapacá. Segunda edición aumentada. Hisbol, La Paz.

Van Kessel, J.

1992b. Cuando Arde el Tiempo Sagrado. Hisbol, La Paz.

Van Kessel, J.

1994 Pachamama, La Virgina: La que creó el mundo y fundó el pueblo. CIDSA, Puno, Tocopilla, Chile.

\section{Notas}

1 Isluga y Cariquima son dos comunidades altiplánicas del norte chileno que se ubican en forma continua a $280 \mathrm{~km}$ al interior de la ciudad de Iquique, en la frontera con Bolivia. Tienen una población aproximada, según Censo 2002, la primera de 1.131 hab., y la segunda de 480 hab. Previo al proceso de regionalización nacional, eran dos comunidades que agrupaban a diferentes pueblos menores o estancias, pero tras este proceso pasaron a ser parte de la actual comuna de Colchane (1.629 hab.). Las actividades económicas más importantes son la ganadería de camélidos (llamas y alpacas) y en menor medida de ovinos, y la agricultura de quinua, papas y ajos en lugares específicos con condiciones más favorables para el cultivo.

2 Jilir Jila: Hermano mayor; Taypir Jila: Hermano del medio; Sullkir Jila: Hermano menor / Jilir Kullaka: Hermana mayor; Taypir Kullaka: Hermana del medio; Sullkir Kullaka: Hermana menor.

3 Las actividades consideradas en la recolección de información fueron: actividades productivas (ganadería; agricultura y textilería); actividades de intercambio (comercio y trabajo); actividades reproductivas (trabajo doméstico); actividades sociales (escuela, comunidad, municipio, rituales). 